\title{
SmellSheet Detective: A Tool for Detecting Bad Smells in Spreadsheets
}

\author{
Jácome Cunha $^{\dagger *}$, João Paulo Fernandes ${ }^{\dagger}$, Pedro Martins ${ }^{\dagger}$, Jorge Mendes ${ }^{\dagger}$, João Saraiva ${ }^{\dagger}$ \\ *Escola Superior de Tecnologia e Gestão de Felgueiras, IPP, Portugal \\ ${ }^{\dagger}$ HASLab / INESC TEC \& Universidade do Minho, Portugal \\ \{jacome,jpaulo,prmartins,jorgemendes,jas\}@di.uminho.pt
}

\begin{abstract}
This tool demo paper presents SmellSheet Detective: a tool for automatically detecting bad smells in spreadsheets. We have defined a catalog of bad smells in spreadsheet data which was fully implemented in a reusable library for the manipulation of spreadsheets. This library is the building block of the SmellSheet Detective tool, that has been used to detect smells in large, real-world spreadsheet within the EUSES corpus, in order to validate and evolve our bad smells catalog.
\end{abstract}

\section{INTRODUCTION}

Spreadsheets play a crucial role in modern society. They are inherently multi-purpose and widely used both by individuals with simple needs as well as by large companies as integrators of complex systems and as support for business decisions. In fact, it is estimated that $95 \%$ of all U.S. firms use them for financial reporting, that $90 \%$ of all analysts in industry perform calculations in them and that $50 \%$ of all spreadsheets are the basis for decisions. Effective mechanisms for error prevention, however, did not grow proportionally: up to $94 \%$ of real-world spreadsheets contain errors [1], which each year cause losses worth around 10 billion dollars!

In this paper we seek to identify potential errors in spreadsheets in an automated way. We look for spreadsheet smells, a concept that was introduced on software by Martin Fowler [2] as a concrete evidence of a possible coding problem. A smell is not necessarily an error, but a characteristic that may cause problems understanding, updating or evolving a software artifact. An example of a software smell is the definition of long methods in an object-oriented program. In the context of spreadsheets, a (bad) smell is, for example, a reference to an empty cell within a spreadsheet formula. An extensive catalog of bad smells for spreadsheets is presented in [3].

To automatically perform the detection of spreadsheets' bad smells we have implemented a library for the analysis of spreadsheets. This library implements our full catalog of smells, that we also review in this paper. The library is reusable and can easily be extended to incorporate further analysis and smells, and it is also the basis for the development of

This work is funded by ERDF - European Regional Development Fund through the COMPETE Programme (operational programme for competitiveness) and by National Funds through FCT - Fundação para a Ciência e a Tecnologia (Portuguese Foundation for Science and Technology) within project FCOMP-01-0124-FEDER-010048. The four first authors were supported by FCT grants SFRH/BPD/73358/2010, SFRH/BPD/46987/2008 and BI4-2011PTDC/EIA-CCO/108613/2008, respectively. Part of this work was developed during a visit to Software Improvement Group (SIG), Amsterdam. the SmellSheet Detective tool. We have used this tool for detecting bad smells in large and real-world spreadsheets, namely by processing the large EUSES spreadsheet corpus to define and evolve our catalog of bad smells. The SmellSheet Detective automatically detects smells given a spreadsheet and the first preliminary results produced are promising: $22 \%$ of the smells automatically detected by our tool are real bad smells, according to a manual validation that we performed. In this paper we present our library and the SmellSheet Detective tool which are the main contributions of this tool paper.

\section{A Catalog of Spreadsheet Smells}

The detection of errors in software systems is an important software engineering research field. Software errors cause programs not to behave as expected and are responsible for several accidents. Even if not necessarily errors, the presence of bad smells in software code can make programs harder to understand, maintain, and evolve, for example. Martin Fowler popularized this notation of program smells in the context of object-oriented programming and this is now an important area of research. The detection of bad smells allows programmers to improve their programs by eliminating them.

In this section we review the catalog of bad smells for spreadsheets introduced in [3]. This catalog was developed using a four steps methodology. In the first step, catalog definition, based on our experience developing and doing research on spreadsheets, an initial catalog of spreadsheets bad smells was proposed. In the second step, catalog validation, in order to validate the catalog we considered a large repository of spreadsheets, the EUSES corpus [4], that contains more than 5000 spreadsheets, and we detected smells in a representative sub-set of the repository: 180 spreadsheets were randomly selected from EUSES which cover the different spreadsheet categories defined in this corpus. In the third step, catalog evaluation, in order to evaluate the results of the empirical experiment performed in the previous step, we manually inspected all the identified bad smells. We classified the detected smells in four categories: not a smell, low smell, medium smell and high smell. In the fourth and last step, catalog refinement, based on the evaluation performed in the previous step, we adjusted the catalog by identifying wrong smells, by refining previously defined smells and by adding new smells that showed up when manually inspecting spreadsheets within the EUSES corpus. The result of this step 
is the catalog of spreadsheets bad smells that we structure in four categories: Statistical Smells (standard deviation smell), Type Smells (empty cell and pattern finder smells), Content Smells (string distance and reference to empty cells smells) and Functional Dependencies Based Smells (Quasi-Functional Dependencies (QFD) smell).

\section{THE SmellSheet Detective TOOL}

In order to automatically analyze sample spreadsheets we implemented SmellSheet Detective ${ }^{1}$ that builds on the above library to detect the smells introduced in Section II. This implementation combines the Java programming language, the Google Web Toolkit (GWT), the Apache POI library and the Google libraries to work with spreadsheets within the Google Docs environment. We decided to support spreadsheets written in the Google Docs platform because it is becoming more and more used, and even the popular Microsoft Office suite has also its online version. Indeed, the migration from desktop to online applications is becoming very common. Nevertheless, we also support spreadsheets written using desktop applications, as can be seen in Figure 1.

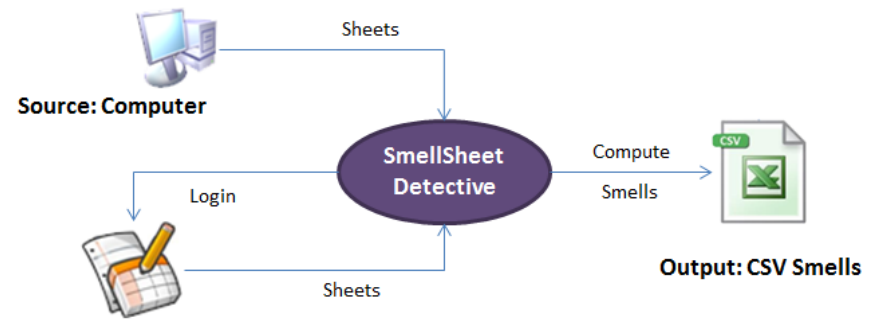

Source: GoogleDocs

Fig. 1. SmellSheet Detective architecture.

When using the Google Docs version of our tool, a valid Google account login is required. After logging in to the account, the tool displays all the spreadsheet files in the account. After choosing a particular spreadsheet file, the user can select a single sheet or the full spreadsheet to be analyzed. If we use the direct upload source, the user can browse the spreadsheets in the computer and select one. After selecting the input, the SmellSheet Detective produces the results of bad smells in several formats (csv, excel and LTEX tables).

Table I presents the results computed by the tool for the 180 spreadsheets considered when evolving our catalog. Each row represents a smell and each column represents the classification we gave to each smell found by the tool. As we can see, $22 \%$ of the smells found are in fact bad smells that deteriorate the quality of the spreadsheet.

The SmellSheet Detective was developed on top of a modular and extensible library written in Java. Next, we present the elegant implementation of the method that computes formulas that contain references to empty cells.

\footnotetext{
${ }^{1}$ Available at http://ssaapp.di.uminho.pt/software/SpreadsheetWA.zip
}

\begin{tabular}{|l|r|r|r|r||r|}
\hline Smell & $\begin{array}{r}\text { Low } \\
\text { smells }\end{array}$ & $\begin{array}{c}\text { Medium } \\
\text { smells }\end{array}$ & $\begin{array}{r}\text { High } \\
\text { smells }\end{array}$ & $\begin{array}{r}\text { Not } \\
\text { smells }\end{array}$ & Total \\
\hline \hline Empty cells & 115 & 4 & 0 & 274 & 393 \\
Patterns & 9 & 5 & 0 & 90 & 101 \\
Std. Dev. cells & 21 & 0 & 0 & 234 & 255 \\
String Dist. & 13 & 2 & 7 & 290 & 312 \\
QFD & 64 & 13 & 6 & 121 & 204 \\
Ref2empty & 9 & 9 & 8 & 24 & 50 \\
\hline Total & 231 & 33 & 21 & 1033 & 1315 \\
\hline
\end{tabular}

TABLE I

RESUlts OF RUNNING SmellSheet Detective IN THE EUSES CORPUS.

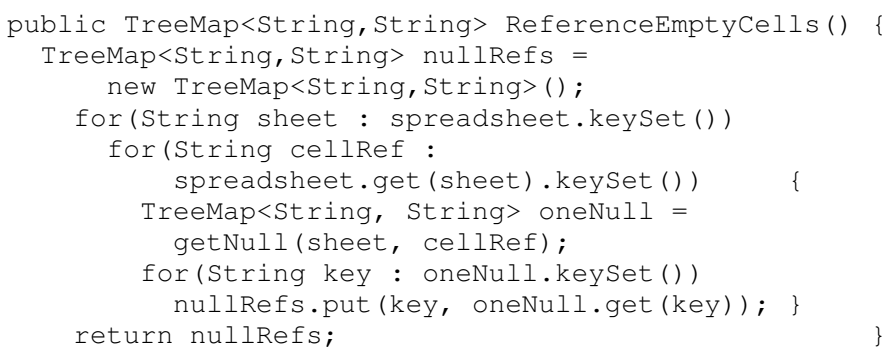

Given the extensibility feature of our library, extending it with new smells is very simple: we just need to add a new method implementing the smell as shown before. This is very important when starting developing our catalog, since new smells can easily been considered. For example, the smells from [5], [6] can easily be incorporated in our tool.

\section{CONCLUSION}

In this paper we have presented the SmellSheet Detective tool and a Java-based library that implements an extensive catalog of bad smells for spreadsheets. The SmellSheet Detective automatically detects bad smells given a spreadsheet as input. We have used it to detect bad smells in the EUSES spreadsheet corpus and the first results are promising. The tool was developed using modular and extensible mechanisms, which allow a simple and efficient implementation of new smells.

Code smells, as suggested by Fowler, are usually associated with refactorings that eliminate them. In fact, we are working now in defining and implementing a set of domain specific spreadsheet refactorings that can be used to eliminate the detected smells.

\section{REFERENCES}

[1] R. Panko, "Facing the problem of spreadsheet errors," Decision Line, 37(5), 2006.

[2] M. Fowler, Refactoring: Improving the Design of Existing Code. Boston, MA, USA: Addison-Wesley, 1999.

[3] J. Cunha, J. P. Fernandes, H. Ribeiro, and J. Saraiva, "Towards a catalog of spreadsheet smells," in 12th Int. Conf. on Computational Science and Its Applications, ser. LNCS, vol. 7336. Springer, 2012, pp. 202-216.

[4] M. F. Ii and G. Rothermel, "The euses spreadsheet corpus: A shared resource for supporting experimentation with spreadsheet dependability mechanisms," in End-User SW Engineering Workshop, 2005, pp. 47-51.

[5] S. Badame and D. Dig, "Refactoring meets spreadsheet formulas," in Proceedings of the 28th IEEE International Conference on Software Maintenance. IEEE Computer Society, 2012, to appear.

[6] M. P. Felienne Hermans and A. van Deursen, "Detecting and visualizing inter-worksheet smells in spreadsheets," in Proc. of the 34rd International Conference on Software Engineering. ACM, 2012, pp. 441-451. 